\title{
Temperature control technology by heat capacity change upon lock and key binding
}

\section{AUTHOR(S):}

Amano, Ken-ichi; Miyazaki, Daisuke; Fong Fong, Liew; Hilscher, Paul; Sonobe, Taro

\section{CITATION:}

Amano, Ken-ichi ...[et al]. Temperature control technology by heat capacity change upon lock and key binding. Physics Letters A 2010, 375(2): 165-169

\section{ISSUE DATE:}

2010-12-26

URL:

http://hdl.handle.net/2433/131851

\section{RIGHT:}

(C) 2010 Elsevier B.V.; This is not the published version. Please cite only the published version.; この論文は出版社版でありません。引用の際に は出版社版をご確認ご利用ください。 


\title{
Temperature Control Technology by Heat Capacity Change upon Lock and Key Binding
}

\author{
Ken-ichi Amano , Daisuke Miyazaki, Liew Fong Fong, Paul Hilscher, \\ and Taro Sonobe \\ Graduate School of Energy Science, Kyoto University, Uji, Kyoto 611-0011, Japan
}

\begin{abstract}
*Corresponding author
E-mail address: k-amano@iae.kyoto-u.ac.jp (K. Amano).
\end{abstract}

\begin{abstract}
We propose an innovative temperature control technology wherein a heat capacity change upon lock and key binding is applied. A proof-of-principle calculation is performed using a three dimensional integral equation theory of a statistical mechanics of fluid (Ornstein-Zernike theory coupled by HNC closure). Logical correctness of the temperature control technology is verified by this calculation. The performance of the heat pump is discussed. In addition, a more effective condition of the heat pump is also discussed to improve the performance.
\end{abstract}

\section{Keywords}

Lock and key principle; Heat pump; Protein; Electrical appliance; $\mathrm{CO}_{2}$ emission. 


\section{Main text}

Currently, variety of temperature control (heating and cooling) technologies exist: vapor compression heat pump [1,2], adsorption refrigerating cycle [3,4], adiabatic demagnetization $[5,6]$, magnetocaloric effect $[7,8]$, and laser cooling $[9,10]$, etc. These technologies are applied as air conditioners or experimental apparatuses. Recently, biological functions are drawn upon in some science areas to create new technologies. For example, water purification and drug synthesis make use of biological functions. In this study, we propose an innovative temperature control technology by drawing upon lock and key principle, a one of biological functions. The proposed temperature control technology is a newly proposed heat pump system which utilizes the heat capacity change upon lock and key binding. A proof-of-principle calculation is performed by using a three dimensional integral equation theory [11-14] of a statistical mechanics of fluid. Logical correctness of the temperature control technology (the heat pump) is verified by the calculation. The performance of the heat pump is the one of the most considerable things. Therefore, the performance of the heat pump is discussed. In addition, a more effective condition of the heat pump is also discussed to improve the performance.

We illustrate the newly proposed heat pump system in Fig. 1. Figure 1 (a) shows a schematic of the heat pump system. There, small spheres forming the solvent exist in the box. The solvent is a simple Lennard-Jones fluid. In the box, a polarized solute (or a solute with partial charges) and a substrate with a pore are immersed. The solute possesses a specific geometric shape that fits into the pore of the substrate. In addition, other suitable shapes for the solute are shown in Fig. 1 (b). Pink and blue represent positive and negative charges, respectively. Although interchanges between the two charges are allowable in these solutes, we explain the heat pump without the interchanges to simplify the explanation. At the outside bottom of the box, either a capacitance or a ferroelectric substance is installed, through which we can apply a positive or negative electric field to the solution. If the condition of the heat pump system is an ideal one, the solute is naturally inserted into the pore due to the lock and key principle [11-19]. However, the condition is not always ideal. In this case, the solute is inserted into the pore by assist of the positive electric field applied from the bottom of the box (i.e., attractive interaction is induced between the solute and the capacitance or the ferroelectric substance). In contrast, when a negative electric field is applied from the bottom of the box, the solute is no longer inserted into the pore. The solute is released from the pore by the electric repulsive interaction. Here, we name the former and the latter states as states A and B, respectively. Switches between states A and B are performed by applying the positive and negative electric fields. States A and B are clearly different from each other. Therefore, solvation structures of states $\mathrm{A}$ and $\mathrm{B}$ are also different. It means that the heat capacities of states A and B are also different (i.e., there is a heat capacity change between states A and B). If judiciously states A and B are switched (detail is shown in next paragraph), the 
temperature of the system can be deliberately controlled by the heat capacity change.

The temperature control cycle is shown in Fig. 2, which utilizes the heat capacity change between states A and B. The heat capacities are under isochoric conditions, because the solution is confined in the (rigid) box. The schema of the temperature control cycle is identical to that of a vapor compression heat pump: It starts from state (I) at room temperature. By applying the positive electric field to the solution at adiabatic condition, the solution changes from state (I) to state (II). The system is cooled. Next, the system is exposed to the atmosphere at room temperature (heat bath). Then, the solution changes from state (II) to state (III). By applying the negative electric field to the solution at adiabatic condition, the solution changes from state (III) to state (IV). The system is heated. Again, the system is exposed to the heat bath, and the temperature of the system returns to room temperature. Then the solution changes from state (IV) to state (I), and the one cycle is completed. By repeating this cycle, the system is functioned as a cooling and/or heating machine.

Next, we show a one possible and practical construction method for the heat pump system (see Fig. 3). The solvent, the solutes, and the substrate with pores are encased in the box, and either a capacitance or a ferroelectric substance is installed at the bottom of the box outside. The size of the box is assumed to be small (millimeter-centimeter size). Several boxes are compiled like Fig. 3, and the temperature control cycle is operated. This is the outline of the temperature control technology we proposed.

To verify the temperature control technology with the new heat pump system, a proof-of-principle calculation is performed. The solvent, the solute, and the substrate with a pore are modeled as follows. Small spheres with diameter $d_{\mathrm{S}}=2.8 \AA$ is the solvent. A large sphere with diameter $d_{\mathrm{U}}=5 d_{\mathrm{S}}$ is the solute. A cylindrical vessel which has $7 d_{\mathrm{S}}$ in inner-diameter is the substrate with a pore. Details of the substrate are shown in Fig. 4. The quasi-Lennard-Jones potential between solvent-solvent $\left(u_{\mathrm{SS}}\right)$ is expressed as

$$
\begin{array}{ll}
u_{\mathrm{SS}}\left(r_{\mathrm{SS}}\right)=\infty & \text { for } r_{\mathrm{SS}}<d_{\mathrm{S}}, \\
u_{\mathrm{SS}}\left(r_{\mathrm{SS}}\right)=-4 \varepsilon\left(d_{\mathrm{S}} / r_{\mathrm{SS}}\right)^{6} & \text { for } r_{\mathrm{SS}} \geq d_{\mathrm{S}} .
\end{array}
$$

$r_{\mathrm{SS}}$ is the distance between the center of the solvent spheres and $\varepsilon$ the interaction constant. $4 \varepsilon /\left(k_{\mathrm{B}} T_{\mathrm{r}}\right.$ ) (dimension less value) is set at 1.5 , and $k_{\mathrm{B}}$ and $T_{\mathrm{r}}$ are the Boltzmann constant and the room temperature of $298.15 \mathrm{~K}$. The quasi-Lennard-Jones potential between solute-solvent $\left(u_{\mathrm{US}}\right)$ is expressed as

$$
\begin{array}{ll}
u_{\mathrm{US}}\left(r_{\mathrm{US}}\right)=\infty & \text { for } r_{\mathrm{US}}<\left(d_{\mathrm{U}}+d_{\mathrm{S}}\right) / 2, \\
u_{\mathrm{US}}\left(r_{\mathrm{US}}\right)=-\xi_{1}\left[d_{\mathrm{S}} /\left(r_{\mathrm{US}}-d_{\mathrm{U}} / 2\right)\right]^{3} & \text { for } r_{\mathrm{US}} \geq\left(d_{\mathrm{U}}+d_{\mathrm{S}}\right) / 2 .
\end{array}
$$


$r_{\mathrm{US}}$ is the distance between the center of the solute and the solvent spheres, $\xi_{1}$ the interaction constant, and $\xi_{1} /\left(k_{\mathrm{B}} T_{\mathrm{r}}\right)$ is set at 0.2 . The quasi-Lennard-Jones potential between substrate-solvent $\left(u_{\mathrm{BS}}\right)$ is expressed as

$$
\begin{array}{ll}
u_{\mathrm{BS}}\left(r_{\mathrm{BS}}\right)=\infty & \text { for } r_{\mathrm{BS}}<d_{\mathrm{S}} / 2, \\
u_{\mathrm{BS}}\left(r_{\mathrm{BS}}\right)=-\xi_{2}\left(d_{\mathrm{S}} / r_{\mathrm{BS}}\right)^{3} & \text { for } r_{\mathrm{BS}} \geq d_{\mathrm{S}} / 2 .
\end{array}
$$

$r_{\mathrm{BS}}$ is the distance between the surface of the substrate and the center of the solvent sphere, $\xi_{2}$ the interaction constant, and $\xi_{2} /\left(k_{\mathrm{B}} T_{\mathrm{r}}\right)$ is set at 0.3 . The potential between solute-substrate $\left(u_{\mathrm{UB}}\right)$ is expressed as

$$
\begin{array}{ll}
u_{\mathrm{UB}}\left(r_{\mathrm{UB}}\right)=\infty & \text { for } r_{\mathrm{UB}}<d_{\mathrm{U}} / 2, \\
u_{\mathrm{UB}}\left(r_{\mathrm{UB}}\right)=0 & \text { for } r_{\mathrm{UB}} \geq d_{\mathrm{U}} / 2 .
\end{array}
$$

$r_{\mathrm{UB}}$ is the distance between the center of the solute sphere and the surface of the substrate. By using above models and the three dimensional integral equation theory, the potential of mean force (PMF) between the solute and the substrate with a pore "in the solvent" can be calculated. Then, the PMF is analyzed to understand the behavior of the solute near the pore. In equation (4), quasi-Lennard-Jones and electrostatic potentials are not set between the solute and the substrate (a capacitance or a ferroelectric substance). However, effects of these disregarded potentials can be straightforwardly superimposed on the PMF calculated with above settings. Therefore, the behavior of the solute near the pore can be predicted even where attractive or repulsive potential is applied between the solute and the substrate (a capacitance or a ferroelectric substance).

A proof-of-principle calculation is performed by using the three dimensional integral equation theory of a statistical mechanics of fluid (Ornstein-Zernike theory coupled by HNC closure) [11-14]. The solute and the substrate with a pore are immersed in the solvent at definite dilution. For the input, $\rho d_{\mathrm{S}}{ }^{3}$ is set at 0.7317 ( $\rho$ is bulk number density of the solvent). The details of the integral equation theory are described in our earlier publications [11-14]. The solute-solvent and the substrate-solvent correlation functions are first calculated from solvent-solvent correlation functions, and then the PMF between the solute and the substrate at temperature $T, \Phi(T ; x, y, z)$, is obtained. Its physical meaning can be understood from

$$
\Phi(T ; x, y, z)=F(T ; x, y, z)-F(T ; \infty, \infty, \infty)
$$

Here, the origin of the coordinate system $(x, y, z)$ is chosen as illustrated in Fig. 4 (b), $F(T ; x, y, z)$ is the Helmholtz free energy of the solution at $T \mathrm{~K}$ where the big sphere is at the position $(x, y, z)$. 
Molar heat capacity change from state B to state A at room temperature at isochoric conditions $\left(\Delta C_{\mathrm{V}}\left(T_{\mathrm{r}}\right)\right)$ is obtained from an equation below.

$$
\Delta C_{\mathrm{V}}(T)=-N_{\mathrm{A}} \cdot T\left[\partial^{2} \Phi(T ; 0,0,0) / \partial T^{2}\right]
$$

where $N_{\mathrm{A}}$ is Avogadro constant. $\Delta C_{\mathrm{V}}$ is the heat capacity change per mol of the pores (solutes). The second order partial differentiation is numerically calculated, which are performed with $\Delta T=1$ K.

In Fig. 5 (a), the PMF between the solute and the substrate at room temperature scaled by $k_{\mathrm{B}} T_{\mathrm{r}}$ on the cross section $z=0, \Phi\left(T_{\mathrm{r}} ; x, y, 0\right) /\left(k_{\mathrm{B}} T_{\mathrm{r}}\right)$, is shown. The PMF only near the pore is shown to visualize the behavior of the solute near the pore. Although an attractive potential between the solute and the substrate is not set, the solute tends to be immersed into the pore in the solvent, for example, through the route indicated by the dots arrow (the route which avoids high potential barriers). After the insertion, it is hard for the solute to move away from the pore due to the high potential barriers. Therefore, the solute is confined in the pore. Similar results have been obtained in articles [11-15]. Figure 5 (b) shows the PMF along the $x$-axis, $\Phi\left(T_{\mathrm{r}} ; x, 0,0\right) /\left(k_{\mathrm{B}} T_{\mathrm{r}}\right)$. The arrow indicates a broad-shelving-slope on the potential curve. Such slope exists also on the potential in the dots arrow. This unnoticeable slope also hinders the insertion of the solute. There is some probability of the insertion, though the insertion does not readily occur in this condition which has no attractive potential between the solute and the substrate (a capacitance or a ferroelectric substance) due to the potential barriers and the slope.

To assist the insertion and the release of the solute into and from the pore, we add negative charges on the solute surface like the rightmost solute in Fig. 1 (b). If sufficiently strong positive electric field is applied to the solution from the bottom of the box, then, the solute is readily inserted into the pore by the electric attractive force. The insertion is facilitated in this way, and the state of heat pump system gets into state A. The solute (with negative charges) is stably confined in the pore even when the positive electric field is eliminated after the insertion (see Fig. 5 (a)). While, sufficiently strong negative electric field is applied to the solution, the solute (with the negative charges) which is confined in the pore is no longer inserted into the pore. The solute is released from the pore by the electric repulsive force, and the state of the heat pump system gets into state B. Thus, switches between states A and B are possible by applying these positive and negative electric fields.

It is important to confirm the existence of the heat capacity change between states A and B in order to perform the temperature control cycle (see Fig. 2). The heat capacity change from state B to state A per mol of the pores (solutes) at room temperature, $\Delta C_{\mathrm{V}}\left(T_{\mathrm{r}}\right)$, is calculated to be -1.3 $\mathrm{kJ} \cdot \mathrm{mol}^{-1} \cdot \mathrm{K}^{-1}$. Although it is small, the existence of the heat capacity change is surely confirmed. Therefore, the temperature can be controlled by using the heat capacity change. 
We have explained that switches between states A and B are possible and showed that the heat capacity change between states A and B surely exists. Therefore, the temperature control technology is logically correct, and the heat pump system is functioned as a cooling and/or heating machine. However, the performance of the heat pump is still unknown. In general, a performance of a heat pump is discussed by means of "coefficient of performance" (COP). It is, however, difficult to discuss using the COP, because we cannot estimate heat (energy) losses of the heat pump. Therefore, we discuss forecasted difference between the hottest and the coldest temperatures of the heat pump to explain its performance. Here, two conditions of the heat pump are considered.

(I) The solute and the substrate with a pore are immersed in 1 mol of the solvent.

(II) Several mmol (mol) of the solutes and the substrate with several mmol (mol) of the pores are immersed in $1 \mathrm{~mol}$ of the solvent.

States A and B of conditions (I) and (II) are schematically shown in Figures 6 and 7. Condition (I) is almost same with the condition set in the calculation, where the solute and the substrate with a pore are immersed in the solvent at infinite dilution. In contrast, condition (II) has so many solutes and pores in the solvent. Condition (II) is clearly different from the condition set in the calculation. States A and B of condition (II) are difficult to treat with theoretical calculations due to its complexity and it is difficult to calculate the hottest and the coldest temperatures. While in condition (I), the hottest $\left(T_{\mathrm{H}}\right)$ and the coldest $\left(T_{\mathrm{C}}\right)$ temperatures can be calculated by using following equations,

$U_{\mathrm{A}}\left(T_{\mathrm{H}}\right)=U_{\mathrm{B}}\left(T_{\mathrm{r}}\right)$,

$U_{\mathrm{B}}\left(T_{\mathrm{C}}\right)=U_{\mathrm{A}}\left(T_{\mathrm{r}}\right)$,

where $U$ is internal energy of the solution, subscripts " $\mathrm{A}$ " and " $\mathrm{B}$ " represent states $\mathrm{A}$ and $\mathrm{B}$, respectively. $U_{i}(i=\mathrm{A}, \mathrm{B})$ is expressed as,

$$
\begin{aligned}
& U_{i}=U_{0}+\Delta U_{i}, \\
& U_{0}=(3 / 2) N_{\mathrm{A}} k_{\mathrm{B}} T+\left(N_{\mathrm{A}} / 2\right) \rho \int_{0}^{\infty} u_{\mathrm{SS}}(r) g_{\mathrm{SS}}(r) \cdot 4 \pi r^{2} d r, \\
& \Delta U_{i}=\Delta \mu_{i}-T\left(\partial \Delta \mu_{i} / \partial T\right)_{\mathrm{V}} .
\end{aligned}
$$

$U_{0}$ is internal energy of the pure solvent (the bulk solvent), $\Delta U$ solvation energy of the solute and the substrate with a pore, $g_{\mathrm{SS}}$ radial distribution function between solvent-solvent, and $\Delta \mu$ solvation free energy of the solute and the substrate with a pore. $\Delta \mu$ is calculated from the 
Singer-Chandler formula [20]. Then, $T_{\mathrm{H}}$ and $T_{\mathrm{C}}$ can be calculated by searching temperatures within equations (7a) and (7b), respectively. However, the difference between these $T_{\mathrm{H}}$ and $T_{\mathrm{C}}$ is thought to be very small (almost zero), because a change of the solvation structure from state B (or A) to state A (or B) is very small (see Fig. 6), which means $\Delta U$ is considerably smaller than $U_{0}$. The performance of the heat pump is not high in condition (I). While in condition (II), the performance of the heat pump is considered to be better than condition (I), because a change of the solvation structure from state B (or A) to state A (or B) is very large (see Fig. 7). The solvation structure drastically changes, so the difference between these $T_{\mathrm{H}}$ and $T_{\mathrm{C}}$ is speculated to be larger than condition (I). This drastic change is very important for the heat pump to improve its performance.

In summary, we have proposed a temperature control technology wherein heat capacity change upon lock and key binding is applied. The proof-of-principle calculation has been performed by using the three dimensional integral equation theory [11-14] of a statistical mechanics of fluid. Logical correctness of the temperature control technology has been verified by the calculation. Also, we examined a variety of adsorbent shapes (not shown here). However, the results indicate that the shape of the pore is important, because the solvation structure and the PMF between the adsorbent and the solute (large sphere) depend on the adsorbent shape. It means that the performance of the heat pump must depend on the adsorbent shape. Although the difference between the hottest and the coldest temperatures of the heat pump has been concluded to be small (low performance) in condition (I), the performance of the heat pump is expected to become higher in condition (II) as compared condition (I). As the next step, we intend to investigate further effective models and conditions.

\section{Acknowledgements}

Basic parts of the computer programs used in the present study were written by Masahiro Kinoshita. This work was supported by the Kyoto University Global Center of Excellence (GCOE) of Energy Science.

\section{References}

[1] F. A. Holland, F. A. Watson, J. Heat Recovery Systems, 2 (1982) 13.

[2] M. V. Rane, K. Amrane, R. Radermacher, Int. J. Refrig. Rev. Int. Froid 16 (1993) 247.

[3] L. Luo, D. Tondeur, Adsorption 6 (2000) 93. 
[4] M. Pons, S. Szarzynski, Int. J. Refrig. 23 (2000) 284.

[5] G. Bernstein, S. Labov, D. Landis, N. Madden, I. Millet, E. Silver, P. Richards, Cryogenics 31 (1991) 99.

[6] A. T. Serlemitsos, M. Sansebastian, E. Kunes, Cryogenics 32 (1992) 117.

[7] K. A. Gschneidner, V. K. Pecharsky, David Fort, Phys. Rev. Lrtt. 78 (1997) 4281.

[8] V. K. Pecharsky, K. A. Gschneidner, Phys. Rev. Lrtt. 78 (1997) 4494.

[9] D. J. Wineland, W. M. Itano, Phys. Rev. A 20 (1979) 1521.

[10] X. Luo, M. D. Eisaman, T. R. Gonsnell, Opt. Lett. 23 (1998) 639.

[11] M. Kinoshita, J. Chem. Phys. 116 (2002) 3493.

[12] M. Kinoshita, T. Oguni, Chem. Phys. Lett. 351 (2002) 79.

[13] M. Kinoshita, Chem. Eng. Sci. 61 (2006) 2150.

[14] K. Amano, M. Kinoshita, Chem. Phys. Lett. 488 (2010) 1.

[15] P. König, R. Roth, Europhys. Lett. 84 (2008) 68006.

[16] L. Mahalakshmi, P. P Das, T. N G. Row, J. Chem. Sci. 120 (2008) 39.

[17] E. J. Petrie, C. S. Clements, J. Lin, L. C. Sullivan, D. Johnson, T. Huyton, A. Heroux, H. L. Hoare, T. Beddoe, H. H. Reid, M. C. J. Wilce, A. G. Brooks, J. Rossjohn, J. Exp. Med. 205 (2008) 725 .

[18] G. Odriozola, F. Jiménez-Ángeles, M. Lozada-Cassou, J. Chem. Phys. 129 (2008) 111101.

[19] S. Sacanna, W. T. M. Irvine, P. M. Chaikin, D. J. Pine, Nature 464 (2010) 575.

[20] S. J. Singer, D. Chandler, Mol. Phys. 55 (1985) 621.

\section{Figure Captions}

Fig. 1. (a) States A and B of the heat pump. Pink and blue represent positive and negative charges, respectively. (b) Other suitable shapes for the solute. Interchanges between these two charges are allowable in these solutes.

Fig. 2. Temperature control cycle. $298.15 \mathrm{~K}$ is the room temperature.

Fig. 3. A one possible and practical construction method for the heat pump system.

Fig. 4. (a) Model of the substrate with a pore. It is colored for three-dimensional visualization. (b) Cross section of the substrate $(z=0)$. The thick solid arrows represent the $x$-axis and $y$-axis. The numbers given are scaled by $d_{\mathrm{S}}$. When a large sphere with diameter $d_{\mathrm{U}}=5 d_{\mathrm{S}}$ (the solute) is inserted into the pore along $x$-axis and contacts the bottom of the pore surface, the center of the solute is at $(x, y)=(0,0)$. 
Fig. 5. (a) The potential of mean force (PMF) between the solute and the substrate with a pore in the solvent at $T_{\mathrm{r}}$ on the cross section $z=0, \Phi\left(T_{\mathrm{r}} ; x, y, 0\right) /\left(k_{\mathrm{B}} T_{\mathrm{r}}\right)$. As the color approaches thick blue, the potential becomes lower, and as the color approaches thick red, the potential becomes higher. The center of the solute can not enter the domain drawn in white. The maximum and minimum values of the potential are also given. These numbers given are scaled by $k_{\mathrm{B}} T_{\mathrm{r}}$. (b) The PMF along the $x$-axis, $\Phi\left(T_{\mathrm{r}} ; x, 0,0\right) /\left(k_{\mathrm{B}} T_{\mathrm{r}}\right)$.

Fig. 6. Schematic illustration of condition (I): the solute and the substrate with a pore are immersed in $1 \mathrm{~mol}$ of the solvent. (a) and (b) represent states A and B, respectively. Green circle and circular arc represent areas where solvation structure is strongly formed.

Fig. 7. Schematic illustration of condition (II): several mmol (mol) of the solutes and the substrate with several mmol (mol) of the pores are immersed in $1 \mathrm{~mol}$ of the solvent. (a) and (b) represent states A and B, respectively. Green circles and circular arcs represent areas where solvation structure is strongly formed. 


\section{State A State B}

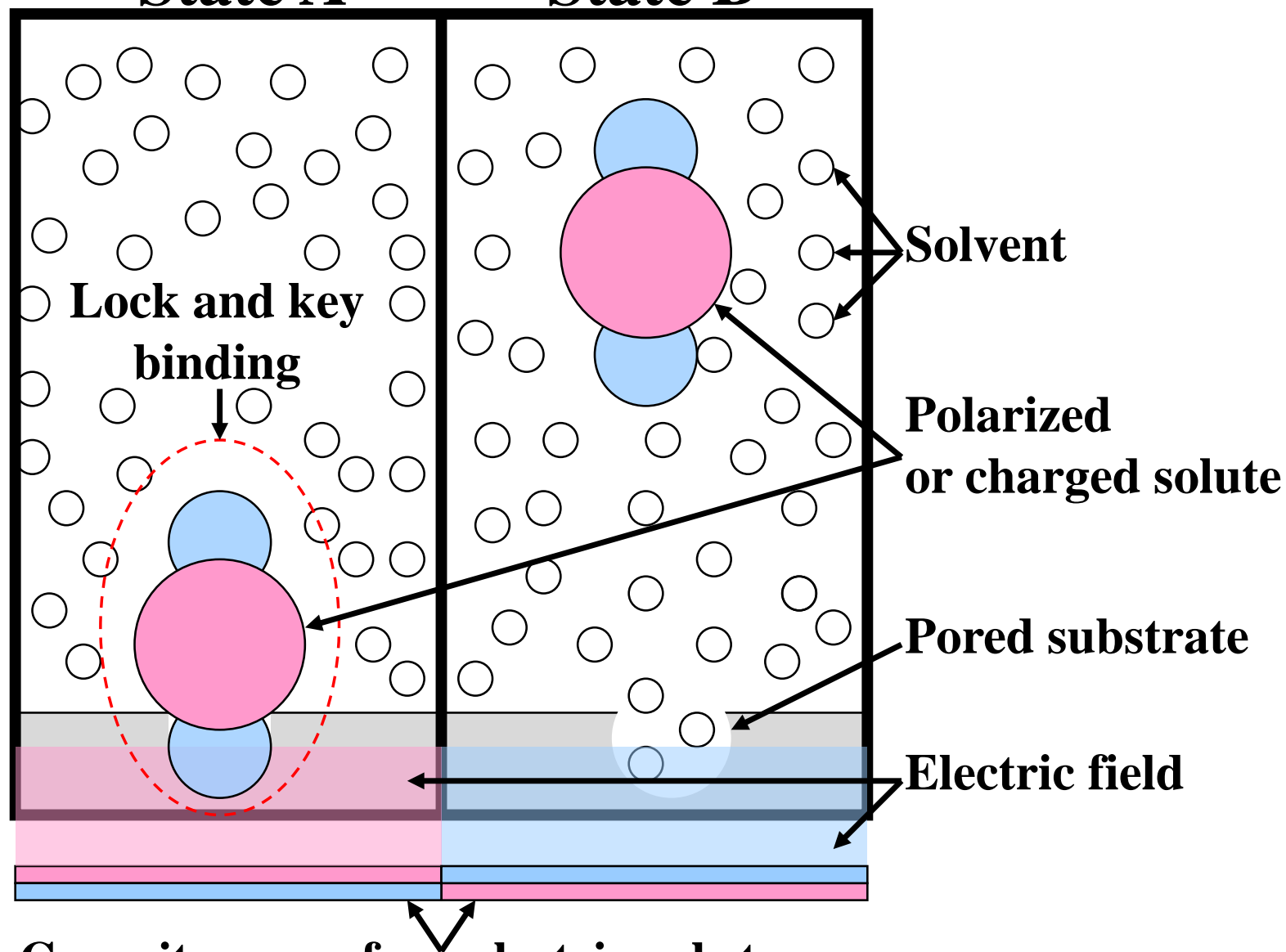

Capacitance or ferroelectric substance

(a)
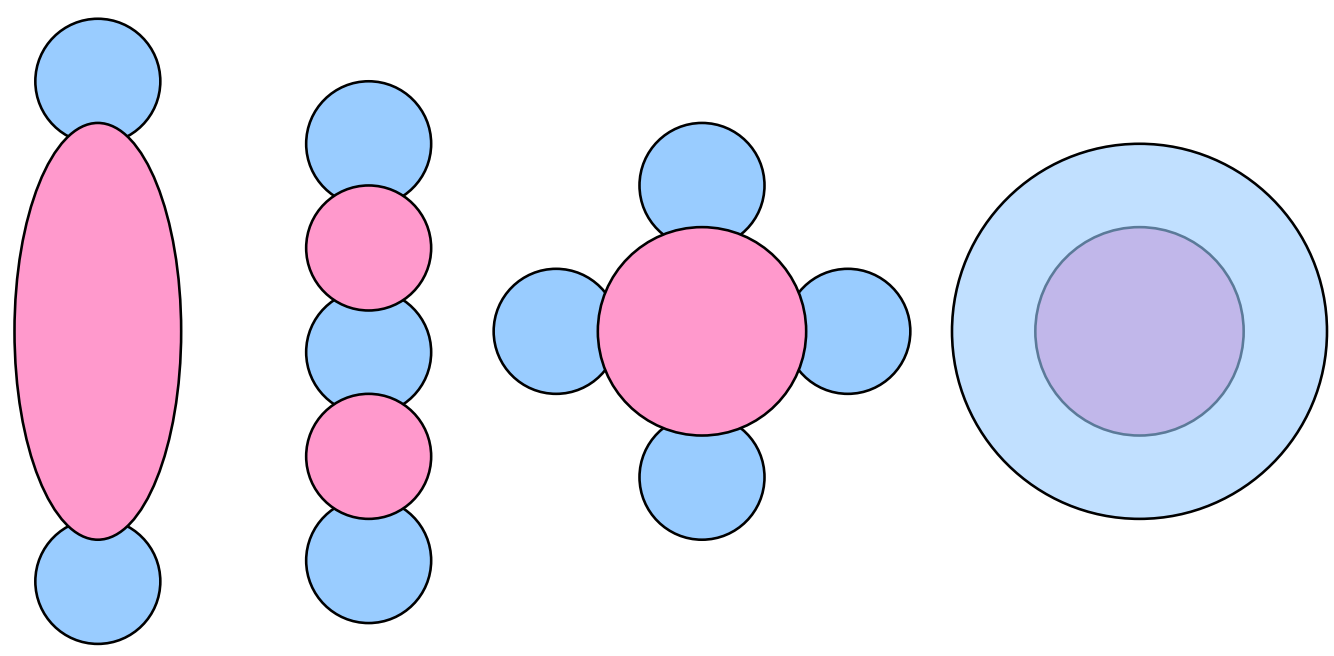

(b)

Fig. 1 


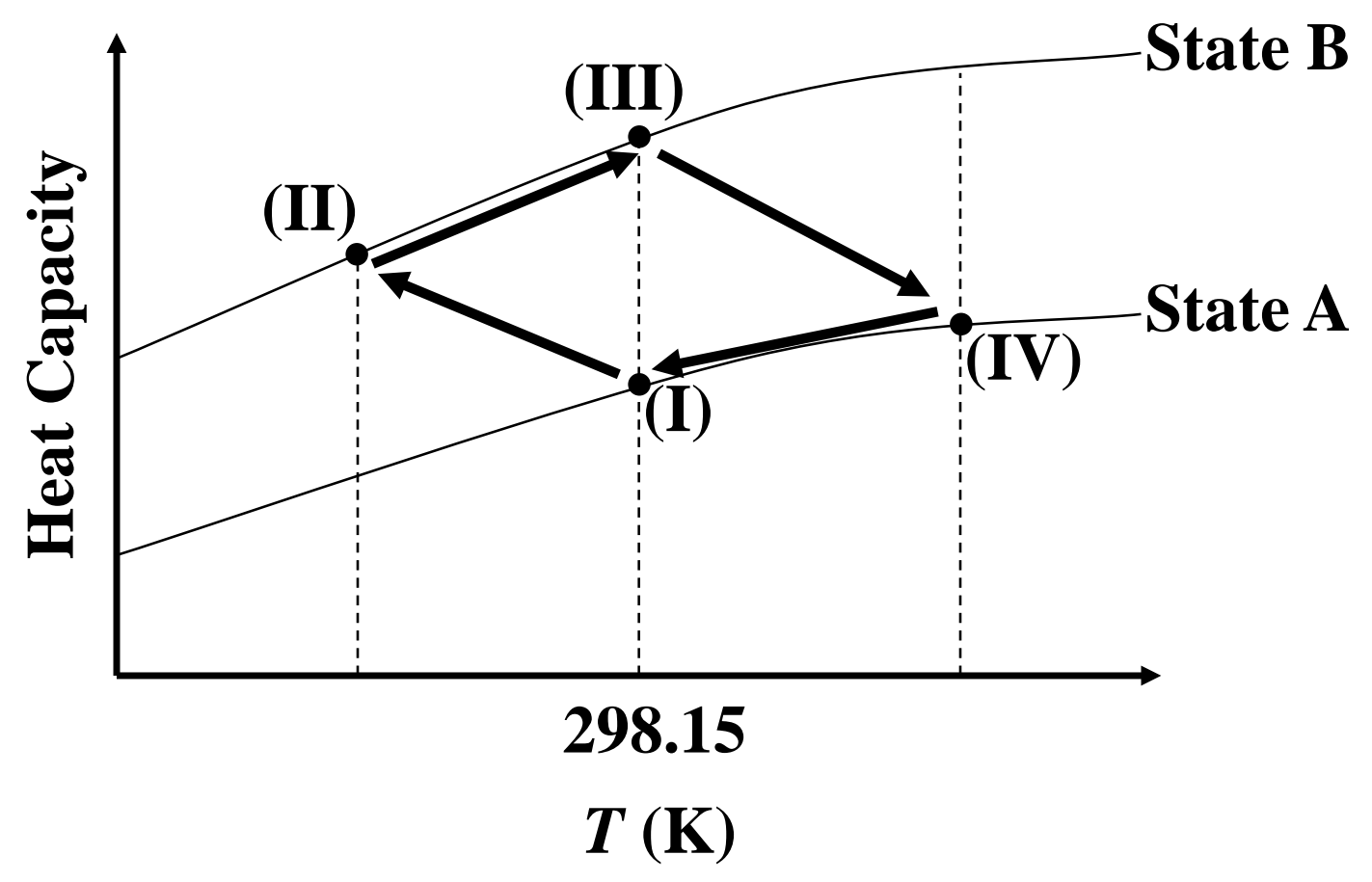

Fig. 2 

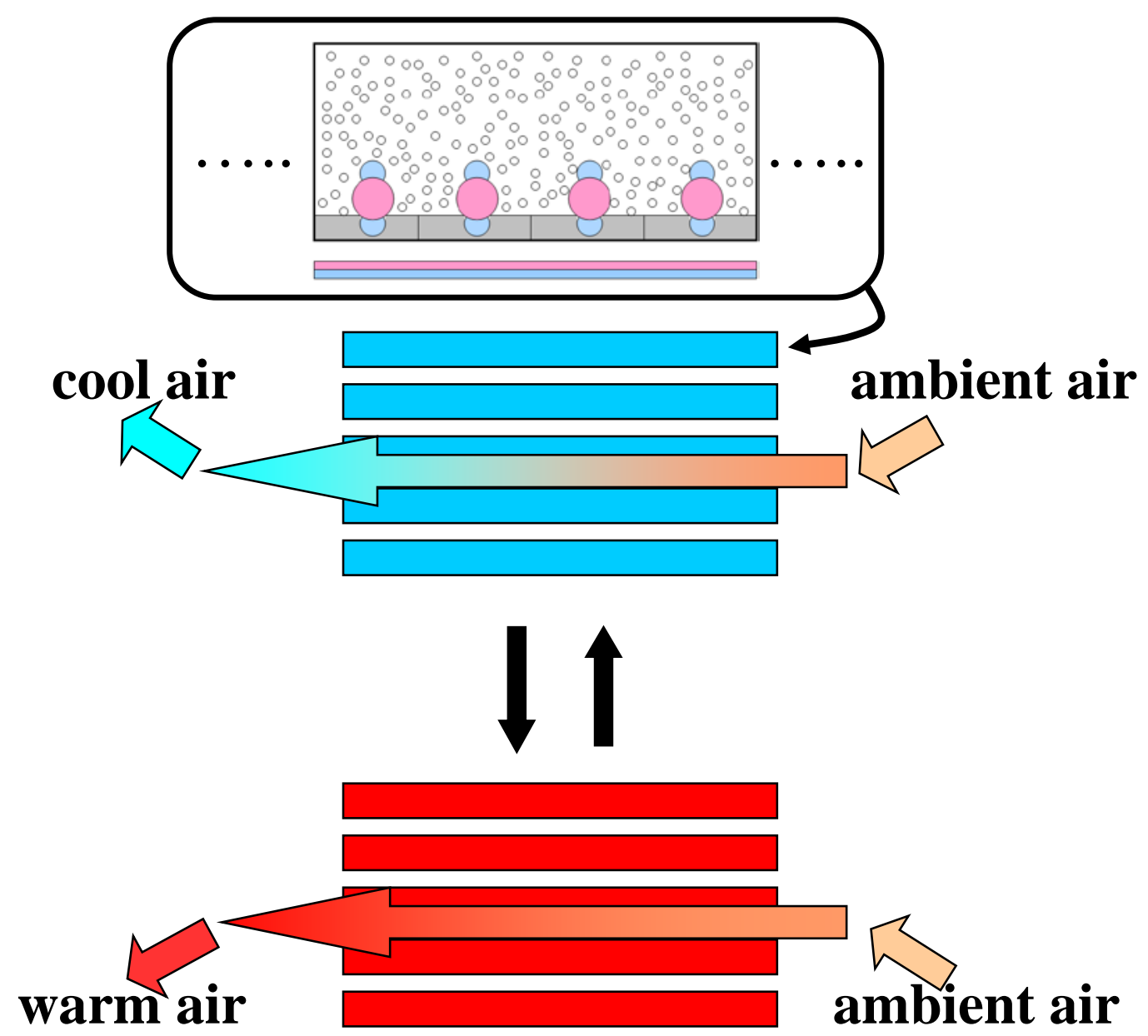

Fig. 3 

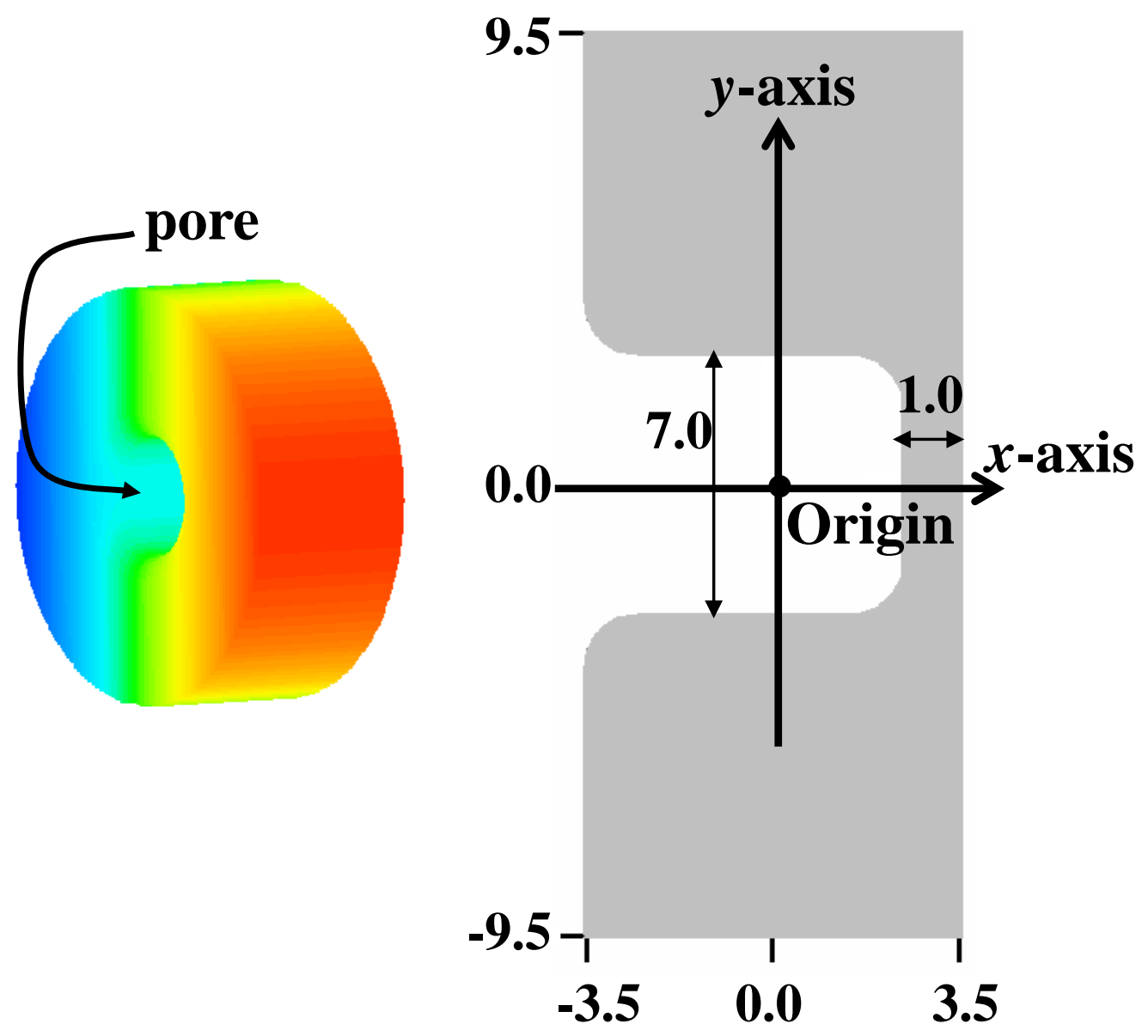

(a)

(b)

Fig. 4 


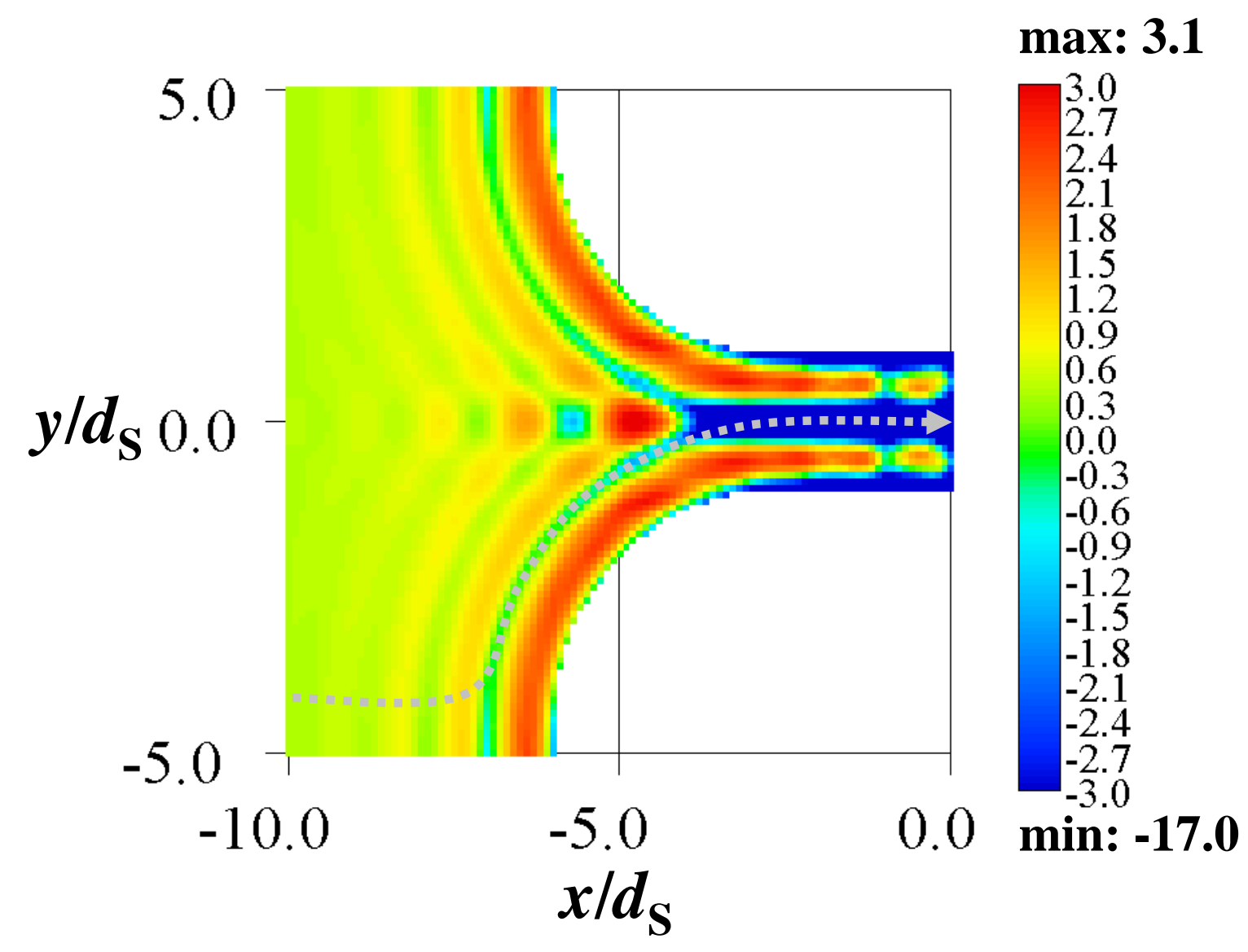

(a)
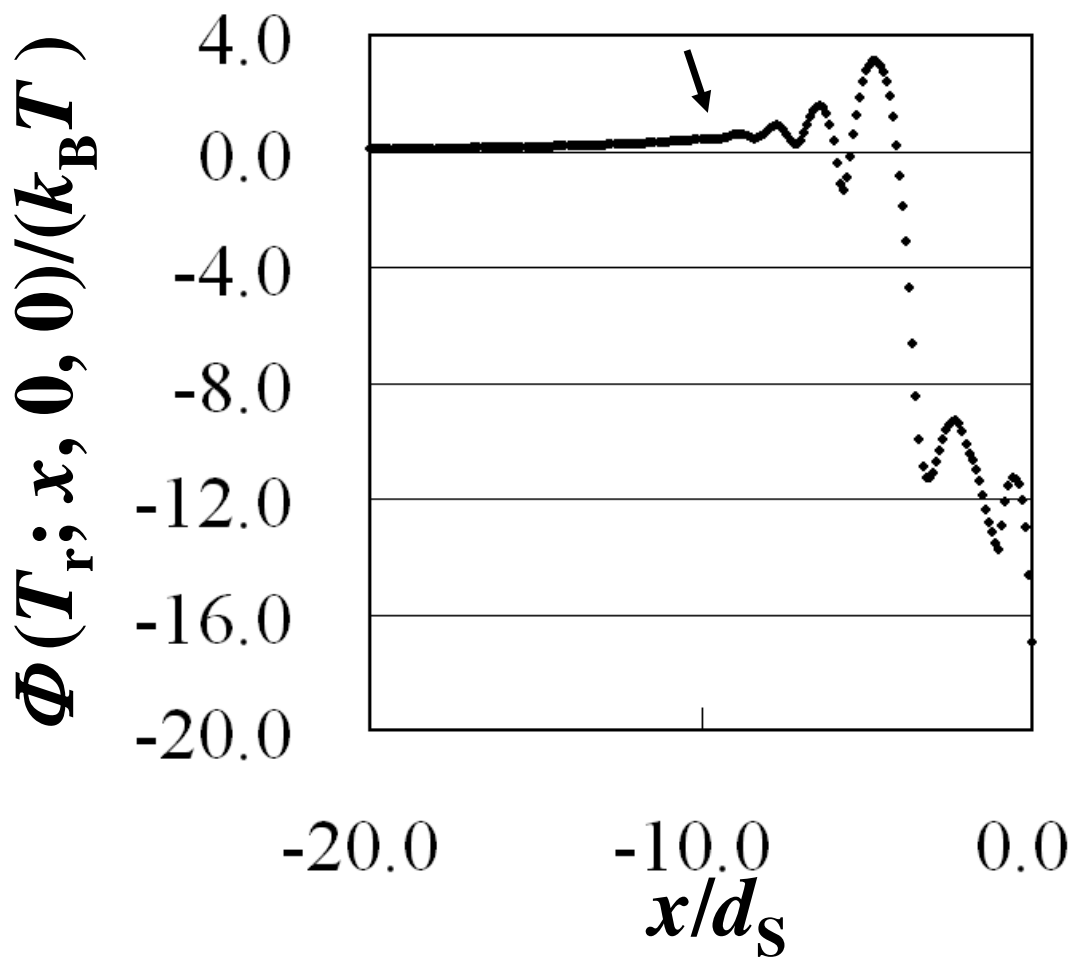

(b)

Fig. 5 


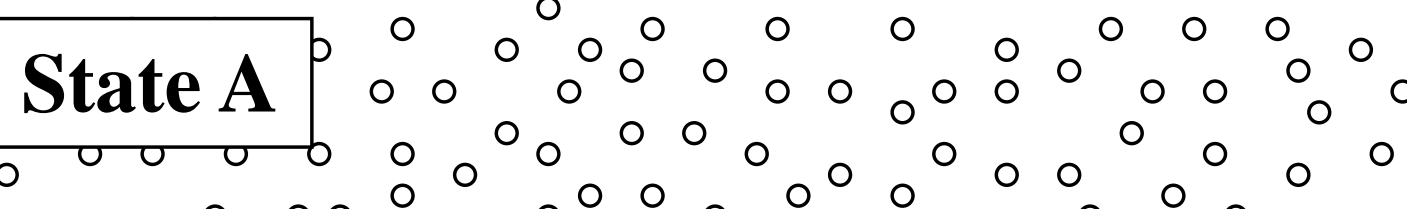

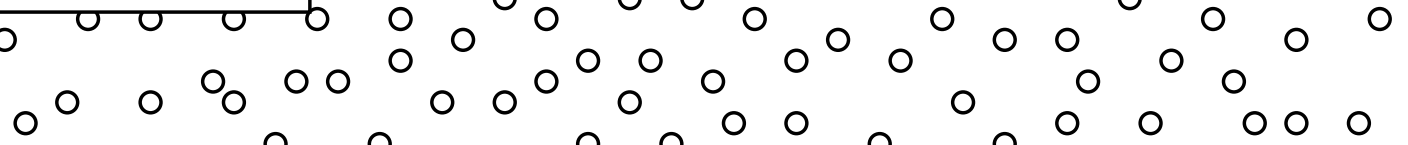

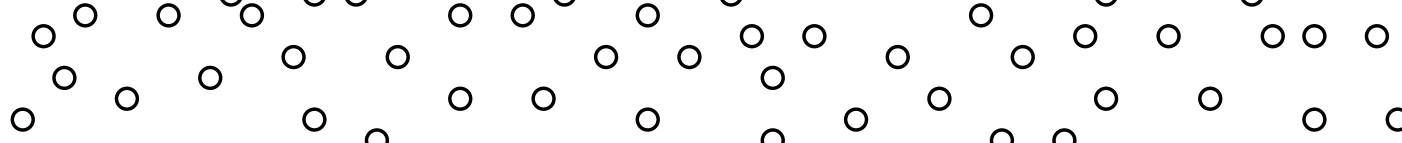

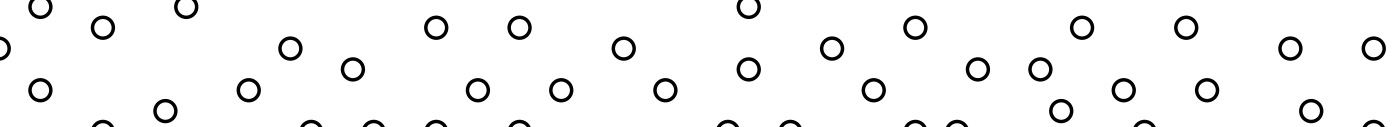

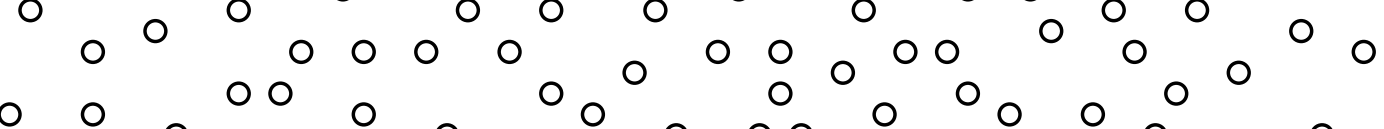

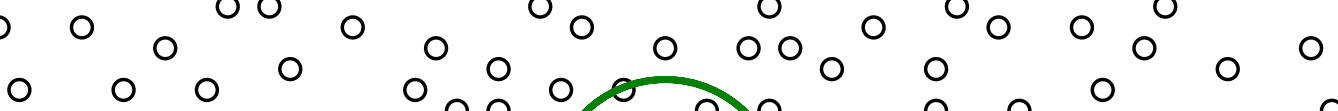

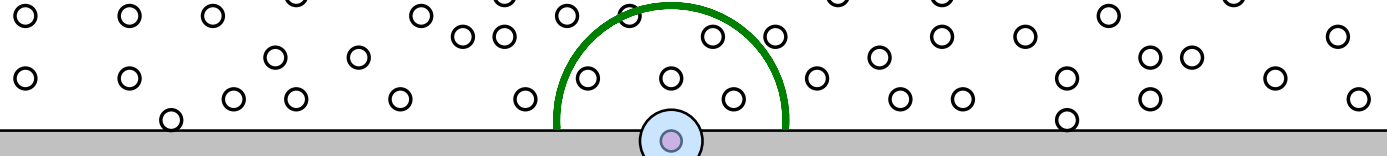

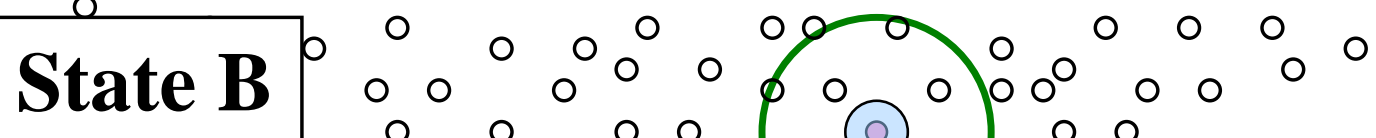

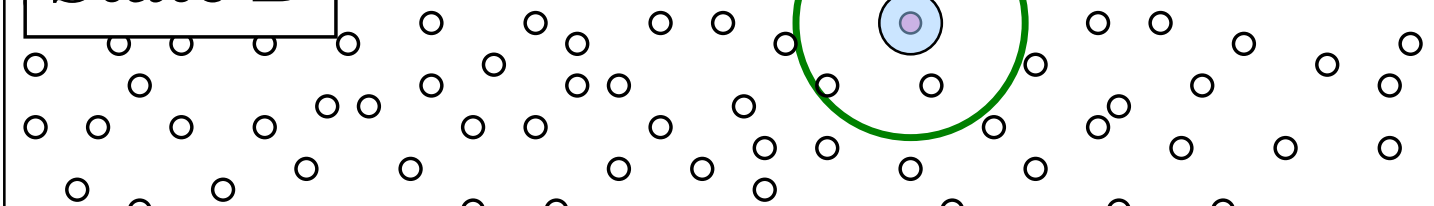

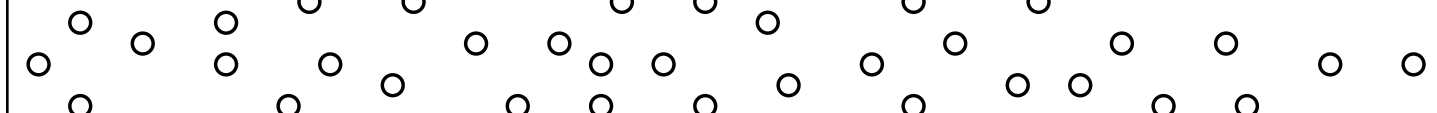

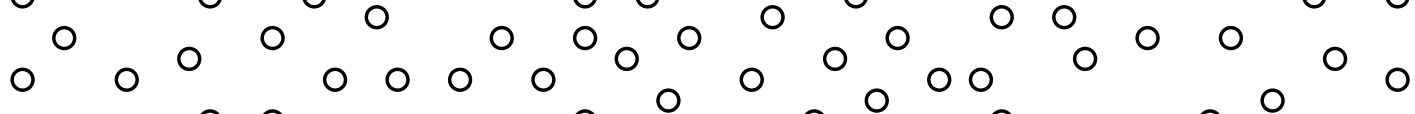

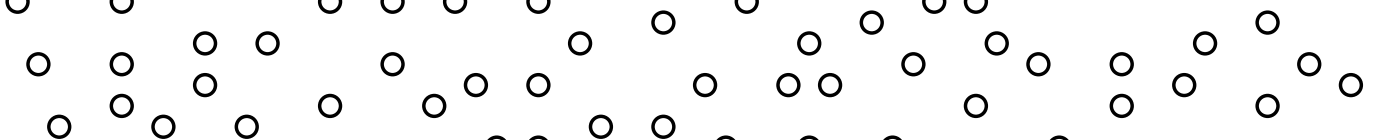

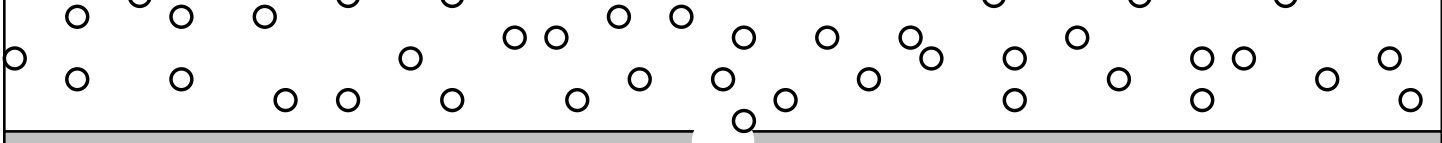




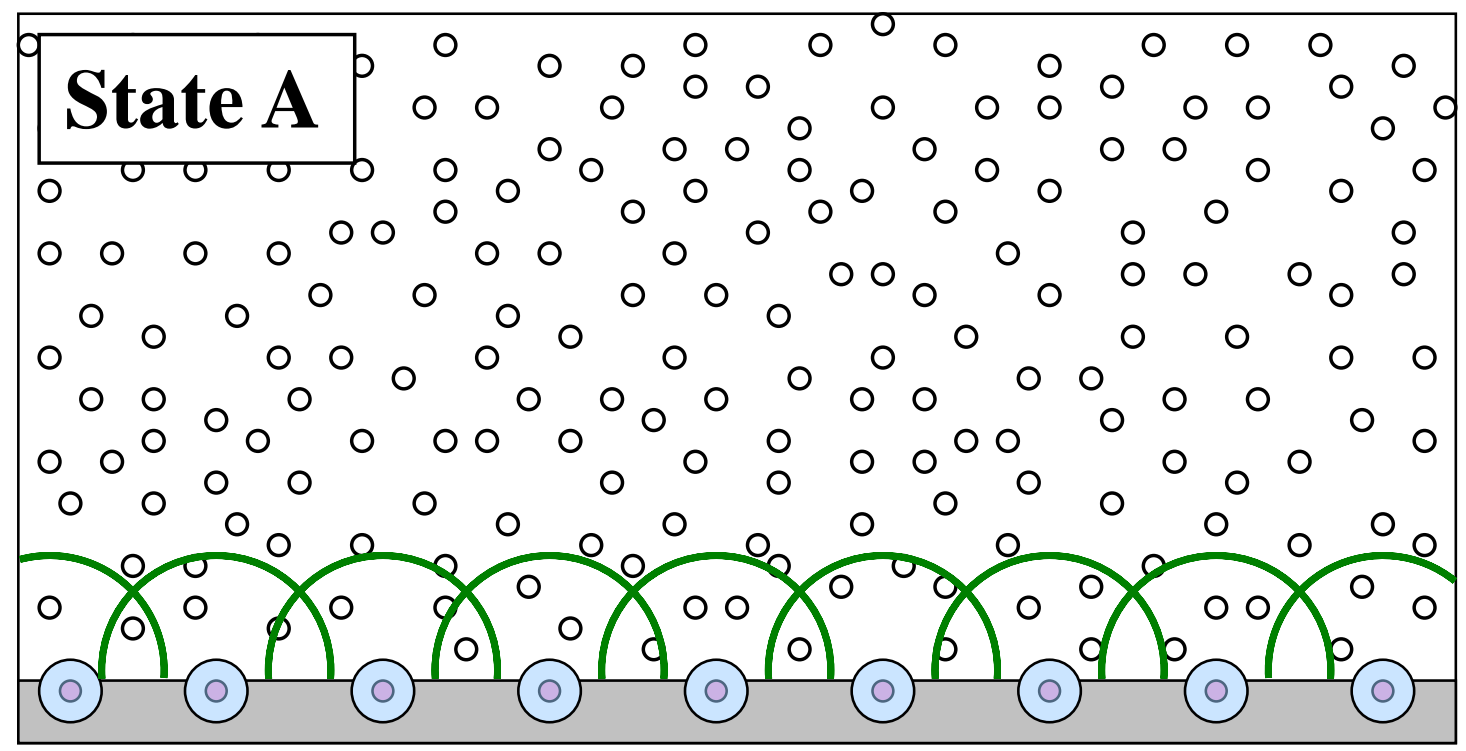

(a)

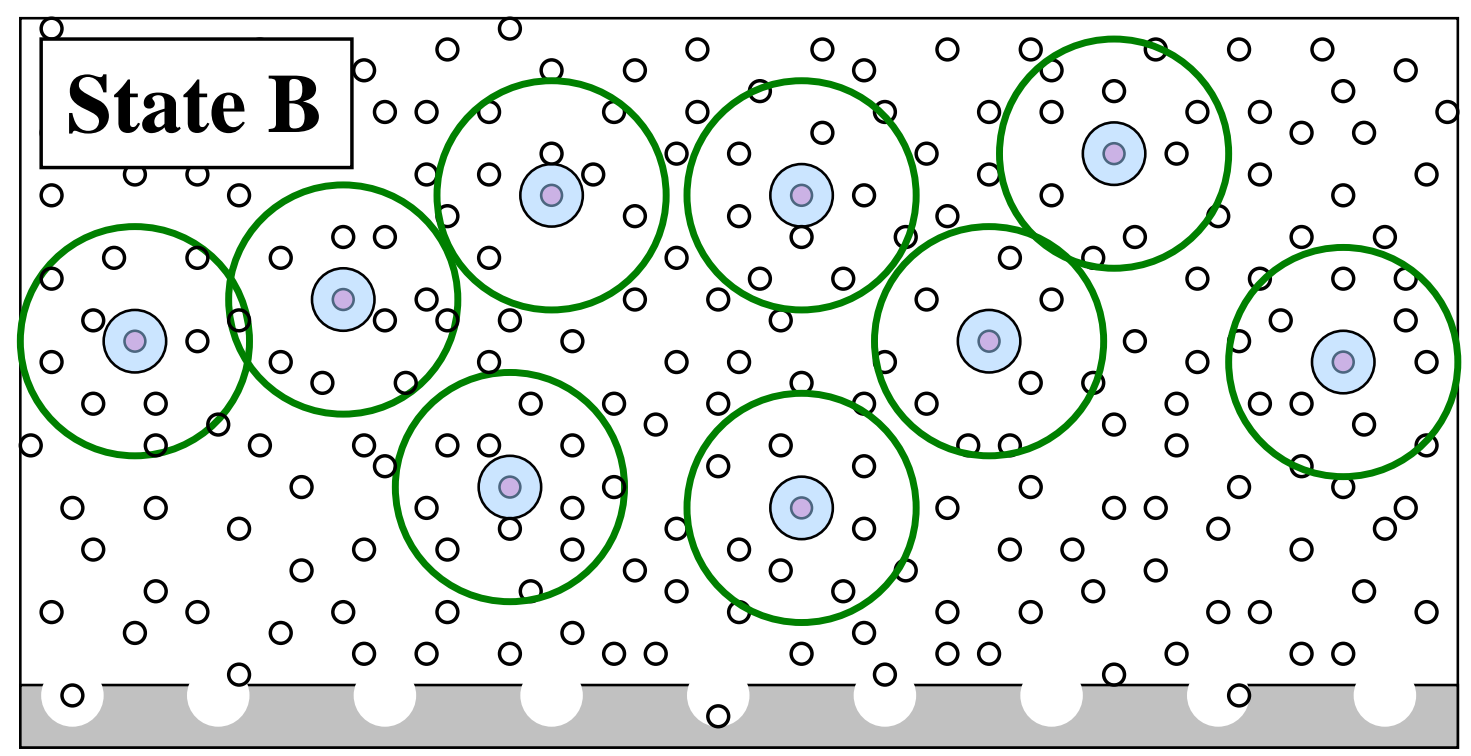

(b)

Fig. 7 